\title{
Effect of Trapped Energetic Particles on the Resistive Wall Mode
}

\author{
G.Z. Hao, ${ }^{1}$ A. K. Wang, ${ }^{1}$ Y. Q. Liu, ${ }^{2, *}$ and X. M. Qiu ${ }^{1}$ \\ ${ }^{1}$ Southwestern Institute of Physics, Post Office Box 432, Chengdu 610041, China \\ ${ }^{2}$ Euratom/CCFE Fusion Association, Culham Science Centre, Abingdon, Oxon OX14 3DB, United Kingdom \\ (Received 6 January 2011; published 1 July 2011)
}

A stability analysis for the resistive wall mode is studied in the presence of trapped energetic particles (EPs). When the EPs' beta exceeds a critical value, a fishbonelike bursting mode (FLM) with an external kink eigenstructure can exist. This offers the first analytic interpretation of the experimental observations [Phys. Rev. Lett. 103, 045001 (2009)]. The mode-particle resonances for the FLM and the $q=1$ fishbone occur in different regimes of the precession frequency of EPs. In certain ranges of the plasma rotation speed and the EPs' beta, a mode conversion can occur between the resistive wall mode and FLM.

It is well-known that future advanced tokamak devices (AT) need to steadily operate in a rather high- $\beta$ ( $\beta=$ $2 \mu_{0} P / B^{2}$, the ratio of the plasma pressure to the magnetic field pressure) region. However, the achievable maximum $\beta$ is often limited by macroscopic MHD instabilities such as the external kink mode. The latter can be completely stabilized by a perfectly conducting wall located close enough to the plasma surface. In a realistic device, the wall has finite conductivity, which only helps to convert the fast growing kink mode to a slowly growing, the so-called resistive wall mode (RWM). The growth time of the RWM is comparable to the wall eddy current decay time. The RWM is unstable in the high- $\beta$ region $\beta_{N}^{\text {no-wall }}<\beta_{N}<\beta_{N}^{\text {ideal }}$, where $\beta_{N}^{\text {no-wall }}$ and $\beta_{N}^{\text {ideal }}$ are the normalized $\beta$ limits without and with an ideal wall, respectively, and $\beta_{N}$ is defined as $\beta_{N}=$ $\beta[\%] a[\mathrm{~m}] B[\mathrm{~T}] / I[\mathrm{MA}]$ with $a, I$, and $B$ being a plasma minor radius, plasma current, and toroidal magnetic field, respectively. Early theories [1-3] have shown that the combination of the plasma flow and certain energy dissipation mechanisms, such as Landau damping, shear Alfvén damping, and classical viscosity, can fully suppress the RWM instability. Further investigations [4-7] have shown that the RWM can be suppressed by a very slow plasma rotation, when the drift kinetic damping, resulting from the resonant interaction between the magnetic precession motion of trapped thermal particles and the mode, is considered. The stabilizing effect of the trapped energetic particles (EPs) on the mode has also been investigated numerically [8-10]. In recent experiments, a fishbonelike bursting mode (FLM) is observed when the neutral beam injection is perpendicularly injected into the high- $\beta$ plasmas $[11,12]$. At the same time, a marginally stable RWM is also observed. We mention that various names for this bursting mode have been proposed in the literature, such as "energetic-particle-driven wall mode (EWM)" in JT-60U [11] and "off-axis fishbone" [12] in DIII-D, respectively.

In this Letter, we explain the above experimental observations, based on an analytic calculation. We use an extended RWM dispersion relation including the contribution of the trapped EPs. We demonstrate that when the perpendicular beta of EPs exceeds a critical value, a FLM instability can be excited, together with the slowly growing RWM.

In what follows, the extended dispersion relation $[4,13]$ of the RWM, neglecting the inertial term but taking into account the contribution of the trapped EPs, is written as

$$
D=-i \omega \tau_{w}^{*}+\frac{\delta W^{\infty}+\delta W_{k}+\delta W_{\mathrm{MHD}, h}}{\delta W^{b}+\delta W_{k}+\delta W_{\mathrm{MHD}, h}}=0,
$$

where $\omega=\omega_{r}+i \gamma$ is the eigenvalue of the RWM instability, with $\omega_{r}$ and $\gamma$ being the real frequency and the growth rate of the mode, respectively. $\delta W^{\infty}$ and $\delta W^{b}$ refer to the perturbed fluid potential energy without and with an ideal wall, respectively. The fluid potential energy includes both the plasma and vacuum contributions. $\delta W_{k}$ and $\delta W_{\mathrm{MHD}, h}$ denote the kinetic and fluid components, respectively, of the perturbed kinetic energy induced by the trapped EPs. The factor $\tau_{w}^{*}=\mu_{0} \sigma b d\left(1-a^{2 m} / b^{2 m}\right) /(2 m)$ is defined as the typical wall eddy current decay time, with $a, b, d$, $m, \sigma$, and $\mu_{0}$ being the plasma minor radius, the wall minor radius, the wall thickness, the poloidal mode number, the wall conductivity, and the permeability of free space, respectively. We note that the fluid energy term (the adiabatic term), associated with EPs, explicitly appears in Eq. (1), but is implicitly included in the total fluid energy in Refs. $[4,13]$.

According to Refs. [14-16], the forms of $\delta W_{K}$ and $\delta W_{\mathrm{MHD}, h}$, in the presence of the plasma flow, can be written as

$$
\begin{aligned}
& \delta W_{k}=-2^{9 / 2} \pi^{3} R m_{h} \int B r d r \int d \alpha \\
& \times \int d E E^{5 / 2} K_{b} \bar{J}^{*} \frac{Q}{\omega-\omega_{0}-\omega_{d}} \bar{J}, \\
& \delta W_{\mathrm{MHD}, h}=-\int d^{3} x\left(\boldsymbol{\xi}_{\perp} \cdot \nabla P_{h \perp}\right)\left(\boldsymbol{\xi}_{\perp}^{*} \cdot \boldsymbol{\kappa}\right),
\end{aligned}
$$

where $\boldsymbol{\xi}_{\perp}, \boldsymbol{\kappa}=(\mathbf{b} \cdot \nabla) \mathbf{b}, P_{h \perp}$, and $m_{h}$ are the perpendicular component of the plasma displacement, magnetic curvature, perpendicular component of the EPs' pressure, and EP mass, respectively; $r$ and $R$ are the radial variable and major radius 
of the torus, respectively; $B=B_{0}(1-\epsilon \cos \theta)$ denotes the equilibrium magnetic field to the lowest order in $\epsilon=r / R$, with $\theta$ being the poloidal angle; $J=\alpha B / 2\left(\nabla \cdot \xi_{\perp}\right)-(1-$ $3 \alpha B / 2) \xi_{\perp} \cdot \boldsymbol{\kappa}$ with $\alpha=\mu m_{h} / E_{k} \quad\left(\mu=v_{\perp}^{2} / 2 B\right.$ and $E_{k}=m_{h} v^{2} / 2 \equiv m_{h} E$ are the magnetic moment and the kinetic energy of EPs, respectively); $Q=\left(\omega-\omega_{0}\right) \partial F /$ $\partial E-(\partial F / \partial r) /\left(r \omega_{c}\right)$ with $F, E$, and $\omega_{c}$ being the distribution function, energy, and cyclotron frequency of the trapped EPs, respectively; $K_{b}$ is defined as $K_{b}=\int_{-\theta_{b}}^{\theta_{b}}(1-$ $\alpha B)^{-1 / 2} d \theta / \pi$, with $\theta_{b}$ being the turning point of the trapped EP. Here we have used $\bar{J}$ to denote the averaging of $J$ over the particle bounce orbit $\left(\bar{J}^{*}\right.$ is the complex conjugate of $\left.\bar{J}\right) ; \omega_{d}$ has been used to express the bounce-average magnetic precession frequency of the trapped EPs, $\omega_{d}=K_{2} E q /$ $\left(K_{b} r \omega_{c} R\right)$, with $q$ being the safety factor and $K_{2}=$ $\int_{-\theta_{b}}^{\theta_{b}}(1-\alpha B)^{-1 / 2} \cos \theta d \theta / \pi=2\left(2 / \epsilon \alpha B_{0}\right)^{1 / 2}\left[2 E\left(k_{t}\right)-\right.$ $\left.K\left(k_{t}\right)\right] / \pi$, where $K\left(k_{t}\right)$ and $E\left(k_{t}\right)$ are the complete elliptic integrals of the first and second kinds, respectively, with the argument $k_{t}=\left(1 / \alpha B_{0}+\epsilon-1\right) /(2 \epsilon)$ [in the following, $E\left(k_{t}\right) \equiv \mathrm{E}$ and $\left.K\left(k_{t}\right) \equiv \mathrm{K}\right] . \alpha B_{0}$ is defined as the pitch angle. In addition, we have used $\omega_{0}$ to denote the Doppler shift frequency.

For simplicity, the external kink mode eigenfunction for a cylindrical equilibrium [13] is used to calculate the quantities $\delta W_{k}$ and $\delta W_{\mathrm{MHD}, h}$. The mode displacement is taken as $\boldsymbol{\xi}_{\perp}=a m \hat{r}^{m-1}\left(\mathbf{e}_{r}+i \mathbf{e}_{\theta}\right) e^{i(m \theta-n \phi)} / F_{0}$ with $n$ being the toroidal mode number, $F_{0}=(m-n q) a /(R q)$, and $\hat{r}=r / a$. From these, the quantity $\bar{J}$ in the $\delta W_{k}$ can be written as $\bar{J}=\left(1-\alpha B_{0} / 2\right) e^{i(-n \phi)} K_{2} m a \hat{r}^{m-1} /\left(2 R F_{0} K_{b}\right)$ with additional assumption of $\nabla \cdot \boldsymbol{\xi}_{\perp}=-2 \boldsymbol{\xi}_{\perp} \cdot \boldsymbol{\kappa}$. Here, we consider the case of $m=2$ and $n=1$. The perpendicular beta of the trapped EPs, averaged over the plasma volume, is given as

$$
\begin{aligned}
\beta_{h} & =\left[\int d^{3} x\left(P_{h \perp}\right) /\left(B^{2} / 2 \mu_{0}\right)\right] / V \\
& =c_{\beta} \int r d r d \alpha d E K_{b} E f,
\end{aligned}
$$

where $c_{\beta}=\pi N_{p} \mu_{0} m_{h} /\left(R B a^{2}\right)$ and $P_{h \perp}=m_{h} \int d^{3} v E F$; $f=2^{5 / 2} R \sqrt{E} F / N_{p}$ is the normalized form of the distribution function, with $N_{p}$ being the total number of EPs.

As for the trapped EPs from the neutral beam injection, we have assumed a slowing-down equilibrium distribution function [i.e., $f \propto E^{-1} \delta\left(a-a_{0}\right)$ ]. The contribution of the trapped EPs to the RWM dispersion relation can be obtained as

$$
\begin{aligned}
\delta \hat{W}_{K 0} \equiv & \delta \hat{W}_{k}+\delta \hat{W}_{\mathrm{MHD}, h} \\
= & 12 \pi\left(1-\frac{\alpha_{0} B_{0}}{2}\right)^{2} \frac{\beta_{h} R}{\mathrm{~K} a}\left\{(\hat{A}-\hat{B}) \frac{2}{7} \Omega \ln \left(1-\frac{1}{\Omega}\right)\right. \\
& -\frac{2}{7}\left(\hat{A}+\frac{5}{2} \hat{B}\right) \Omega\left[2\left(\frac{1}{5 \Omega}+\frac{1}{3 \Omega^{2}}+\frac{1}{\Omega^{3}}\right)\right. \\
& \left.\left.-\frac{1}{\Omega^{3}} \frac{1}{\sqrt{\Omega}} \ln \left(\frac{1+\sqrt{\Omega}}{1-\sqrt{\Omega}}\right)\right]\right\}+M
\end{aligned}
$$

where $\quad \delta \hat{W}=2 R \mu_{0} F_{0}^{2} \delta W /\left(\pi B^{2} a^{4} m^{2}\right), \quad$ and $\quad \Omega=$ $\left(i \gamma+\omega_{r}-\omega_{0}\right) / \omega_{d s} \equiv i \gamma / \omega_{d s}+\Omega_{r}-\Omega_{0}$.

The coefficients are

$$
\begin{aligned}
\hat{A}= & (2 m-2)(2 \mathrm{E}-\mathrm{K}) / q+(2 \mathrm{E}-\mathrm{K}) /(2 q) \\
& -(2 \mathrm{E}-\mathrm{K}) /\left(\alpha_{0} B_{0}\right)(\mathrm{E} / \mathrm{K})^{\prime}-\left[\mathrm{E} /\left(\alpha_{0} B_{0} \mathrm{~K}\right)\right. \\
& \left.+k_{t} / q-1 /\left(2 \alpha_{0} B_{0}\right)-1 /(2 q)\right](2 \mathrm{E}-\mathrm{K})^{\prime},
\end{aligned}
$$

$\hat{B}=\left[\left(1-2 k_{t}\right) / q-(2 \mathrm{E} / \mathrm{K}-1) /\left(\alpha_{0} B_{0}\right)\right] \mathrm{K}(\mathrm{E} / \mathrm{K})^{\prime}$

$$
-(2 \mathrm{E}-\mathrm{K}) / q \text {, }
$$

$M=-12 \pi\left(1-0.5 \alpha_{0} B_{0}\right)^{2} \beta_{h} R\left(1-2 k_{t}\right)(1-1 / q)$

$$
\times(2 \mathrm{E}-\mathrm{K})^{\prime} /[\mathrm{K} a(4 m-3)],
$$

and $\omega_{d s}=K_{2}(a) E_{m} q /\left[K_{b}(a) m_{h} a \omega_{c} R\right]$ denotes the precession frequency of the trapped EPs at the plasma edge, with $E_{m}$ being the birth energy of the trapped EPs. The prime in Eqs. (6)-(8) denotes the derivation with respect to $k_{t}$. To obtain a simple expression of $\delta \hat{W}_{K 0}$, in the above calculation, we have assumed that the arguments of elliptic integrals are independent of the minor radius $r$. These elliptic integrals are approximated by their edge values. Numerical calculations without this approximation do not show qualitatively different results. The form of $\delta \hat{W}_{K 0}$ is analogous to that of Eq. (29) in Ref. [15]. Equation (5) shows that, at the marginal stability (i.e., $\gamma=0$ ), either $\Omega \leq 1$ or $\Omega>1$ can induce a finite $\operatorname{Im}\left(\delta \hat{W}_{K 0}\right)$ (the imaginary part of $\delta \hat{W}_{K 0}$ ) through the resonance between the trapped EPs and the mode. The resonances of the cases $\Omega \leq 1$ and $\Omega>1$ are embedded in the terms $\ln (1-1 / \Omega)$ and $\ln [(1+\sqrt{\Omega}) /(1-\sqrt{\Omega})]$, respectively. We mention that an analytic calculation has been made for the $(m=$ 2, $n=1$ ) mode in Ref. [17], where a fast growing instability, with double kink structure, is predicted as the hot ion beta exceeds a threshold value. However, the above cited work is based on an ideal kink dispersion relation, and with a special choice of the top-hat structure for the mode eigenfunction. In this Letter, we use the RWM dispersion relation, and assume an external kink mode eigenfunction.

According to Ref. [13], the normalized forms of the fluid potential energy without and with an ideal wall are given, respectively, as

$$
\delta \hat{W}^{\infty}=-4 \pi(m-n q)^{2}[1 /(m-n q)-1] /\left(m q^{2}\right)
$$

and

$$
\begin{aligned}
\delta \hat{W}^{b}= & -4 \pi(m-n q)^{2}\{1 /(m-n q) \\
& \left.-1 /\left[1-(b / a)^{-2 m}\right]\right\} /\left(m q^{2}\right) .
\end{aligned}
$$

Combining Eq. (5) with Eqs. (9) and (10), we obtain the normalized dispersion relation

$$
\begin{aligned}
D= & \delta \hat{W}^{b}+\left(\delta \hat{W}^{\infty}-\delta \hat{W}^{b}\right) /\left[1-i\left(\Omega_{r}+i \gamma / \omega_{d s}\right) \omega_{d s} \tau_{w}^{*}\right] \\
& +\delta \hat{W}_{K 0}=0 .
\end{aligned}
$$

When the effect of trapped EPs is taken into account, the eigenvalue of RWM should be modified according to 
Eq. (11). It can be noted that, in Eq. (11), $\delta \hat{W}_{K 0}$ is calculated for a large aspect ratio toroidal plasma, while $\delta \hat{W}^{\infty}$ and $\delta \hat{W}^{b}$ are calculated for a cylindrical plasma. The toroidal effect will change the fluid potential energy. We have performed a numerical sensitivity study by varying the values of the fluid energy in a wide range, and found that the final conclusions still hold. The plasma shaping (e.g., elongation and triangularity) can also affect the results, mainly by changing the mode eigenfunction and the trapped particle precession frequency. The change of the mode eigenfunction modifies both the fluid and the drift kinetic energy perturbations. The modification of the trapped particle precession frequency has been shown to reduce the hot ion pressure threshold for triggering the $q=1$ fishbone [18]. The plasma shaping effects are not considered in this work.

We solve the dispersion relation (11) numerically, choosing the following parameters: $m=2, n=1, a=1 \mathrm{~m}$, $R=3 \mathrm{~m}, B_{0}=2.3 \mathrm{~T}, E_{m}=85 \mathrm{KeV}, q=1.42, \beta=$ $0.055, b=1.2 a, \sigma=10^{6} \Omega^{-1} \mathrm{~m}^{-1}, d=0.01 a$, and the density $n_{0}=10^{20} \mathrm{~m}^{-3}$. These operating conditions are chosen, mainly to have a fluid unstable RWM regime in the absence of kinetic effects. We also assume a flat $q$ profile to simplify calculations. The safety factor enters into the drift kinetic energy only via the plasma perpendicular displacement (the mode eigenfunction). A separate investigation has been carried out, in order to understand how the variation of the mode eigenfunction changes our drift kinetic analysis. In particular, by considering the $m / n=3 / 1$ mode, we find that the fishbonelike instability can still occur when $\beta^{*}$ exceeds a threshold value.

Figure 1 plots the normalized eigenvalue versus $\beta^{*} \equiv$ $\beta_{h} / \beta$. Figure 1(a) shows that when $\beta^{*}$ is less than the critical value $\beta_{c}^{*}=0.141$, only one branch of instability (the unstable RWM with the damping effect of the trapped EPs) exists. However, when $\beta^{*}>\beta_{c}^{*}$, there are two unstable branches: one branch (solid curve) is completely suppressed when $\beta^{*}>0.2$. This is the conventional RWM branch. Rotational stabilization of this branch is achieved solely by the mode resonance with EPs in our model, not by other damping mechanisms such as the mode resonance with the Alfvén or sound wave continua. The other branch (dashed curve) is a bursting mode (i.e., FLM), with initial real mode frequency $\Omega_{c}=0.75$, and the mode growth rate $\gamma \tau_{w}^{*}$ being roughly a linear function of $\beta^{*}\left(>\beta_{c}^{*}\right)$. At $\beta^{*}=0.2$, the real frequency of the FLM is $\omega_{r}=2.6 \omega_{d s}=1.5 \times 10^{4} \mathrm{rad} / \mathrm{s}^{-1}$, or $f_{r}=2.5 \mathrm{kHz}$, as shown by Fig. 1(b), and the mode growth rate $\gamma=$ $2.8 \times 10^{3} \mathrm{~s}^{-1}$. These values are qualitatively comparable with the experimental results of $f_{r}^{\exp } \approx 3.0 \mathrm{kHz}$ and $\gamma^{\exp } \approx$ $1.0 \times 10^{3} \mathrm{~s}^{-1}$ [11]. Reference [11] also shows that the bursting mode has a 2/1 structure peaking near the $q=2$ rational surface, and it has a $3 / 1$ structure at the plasma edge.

Figure 1 also shows a possible mode conversion between the RWM and the FLM. In fact, at a certain value of the plasma rotation speed $\left(\Omega_{0}=-0.76\right.$ for the case considered here), the two branches of solution can merge, as shown by the dotted curves in Fig. 1. For our case, the direct mode conversion can approximately occur in a region of $0.12<$ $\beta^{*}<0.14$, where the mode possesses both the RWM character (growth rate decreasing with $\beta^{*}$ ) and the FLM character (real frequency increasing with $\beta^{*}$ ).

Figure 2 displays $\operatorname{Im}\left(\delta \hat{W}_{K 0}\right)$ as a function of $\beta^{*}$. It is well-known that the term $\operatorname{Im}\left(\delta \hat{W}_{K 0}\right)$ is always stabilizing for the RWM [4]. For the conventional RWM branch (solid curve $), \operatorname{Im}\left(\delta \hat{W}_{K 0}\right)$ increases with $\beta^{*}$; hence, the mode growth rate decreases with increasing $\beta^{*}$. On the contrary, the value of $\operatorname{Im}\left(\delta \hat{W}_{K 0}\right)$ for the FLM branch (dashed curve) decreases with increasing $\beta^{*}$, and hence the FLM growth rate increases with $\beta^{*}$, as shown in Fig. 1. The dotted curve in Fig. 2 shows a situation when a direct mode conversion occurs between the RWM and the FLM branches.

It is worth noting that, at critical point $\beta_{c}^{*}$, the Doppler shifted real frequency for the FLM is denoted by $\Omega=$ $\Omega_{c}-\Omega_{0}>1$, which can result in a finite $\operatorname{Im}\left(\delta \hat{W}_{K 0}\right)$ due to the term $\ln [(1+\sqrt{\Omega}) /(1-\sqrt{\Omega})]$ in Eq. (5). Physically, the finite $\operatorname{Im}\left(\delta \hat{W}_{K 0}\right)$ arises from the resonant interaction between the trapped EPs and mode [i.e., $\Omega_{c}-\Omega_{0}-$ $\left.\omega_{d}\left(r_{s}\right) / \omega_{d s}=0\right]$, occurring at $r=r_{s}<a$ the plasma minor radius. Because of the dominant $1 / r$ dependence of the precession frequency, the resonant condition can always be satisfied at a certain minor radius. For the case without the plasma rotation $\left(\Omega_{0}=0\right)$, the similar fishbonelike branch $\left(\Omega_{c}>1\right)$ can also be found, with the conventional RWM branch having a considerably higher growth rate, due to the lack of the stabilization from the plasma rotation. We must point out that the dominant kinetic contribution from the EPs, for the FLM excitation, comes from a resonance term such as $\ln [(1+\sqrt{\Omega}) /(1-\sqrt{\Omega})]$, while the resonance for the classical $q=1$ fishbone mode, following White-Chen et al. [14,15], is mainly associated with the $\ln (1-1 / \Omega)$-like term. Both resonances are associated
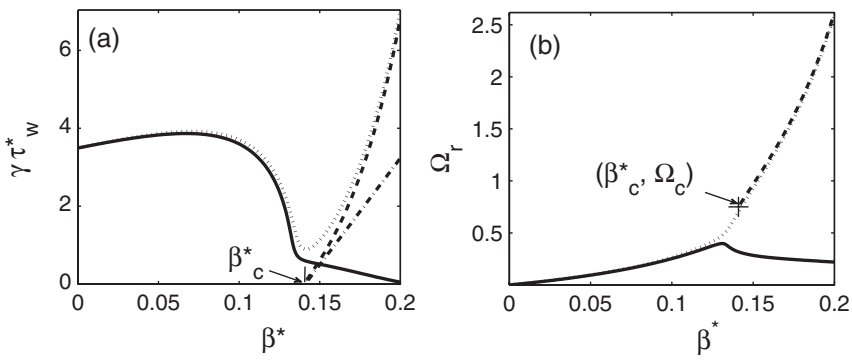

FIG. 1. The (a) normalized growth rate $\gamma \tau_{w}^{*}$ and (b) mode frequency of the conventional RWM mode (solid curve) and the FLM (dashed curve) as functions of the trapped EPs' $\beta^{*}$, for the particle pitch angle $\alpha_{0} B_{0}=0.95$ and the normalized plasma rotation frequency $\Omega_{0}=-0.73$. The dotted curve corresponds to the case of $\Omega_{0}=-0.76$. The minus sign of $\Omega_{0}$ implies that the plasma flow is in the opposite direction of the EPs' magnetic precession. The dash-dotted curve in (a) shows the growth rate from a linear perturbative analysis. The + symbol in (b) indicates the marginal point for the FLM excitation. 


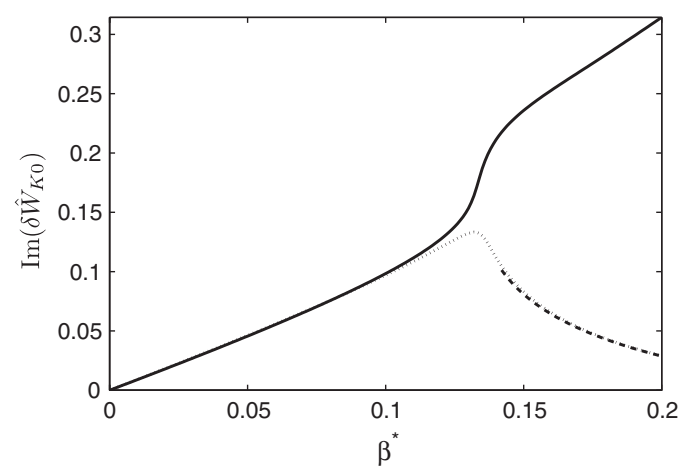

FIG. 2. The imaginary part $\operatorname{Im}\left(\delta \hat{W}_{K 0}\right)$ of the perturbed energy of the trapped EPs versus the trapped EPs' $\beta^{*}$, with the chosen parameters $\alpha_{0} B_{0}=0.95$ and $\Omega_{0}=-0.73$, for the conventional RWM (solid curve) and the FLM (dashed curve) branches. The dotted curve corresponds to the case of $\Omega_{0}=-0.76$.

with the magnetic precession of EPs, but occurring in different regimes. The value of EPs' beta threshold ( $\equiv \beta_{c}^{*} \beta$ ) for the $q=1$ fishbone is about 0.002 [15]. This threshold value is about 0.0077 for the FLM in our study, at $\Omega_{0}=-0.73$.

Equation (11) can be solved, following a standard perturbation expansion method, near the marginal point $\left(\beta_{c}^{*}, \Omega_{c}\right)$ for the FLM, by writing $\Omega=\Omega_{c}+\delta \Omega_{c}+i \gamma / \omega_{d s}$ and $\beta^{*}=\beta_{c}^{*}+\delta \beta^{*}$. Here, the values of $\Omega_{c}$ and $\beta_{c}^{*}$ are obtained by solving the equations $\operatorname{Im}\left[D\left(\Omega_{c}\right)\right]=0$ and $\operatorname{Re}\left[D\left(\Omega_{c}\right)\right]=0$. Linearizing the dispersion relation (11), and taking into account the condition $\Omega_{c} \omega_{d s} \tau_{w}^{*} \gg 1$, valid for the FLM, we obtain the following equation for the perturbed growth rate of the FLM near the marginal point $\beta_{c}^{*}$ :

$$
\gamma \tau_{w}^{*}=\frac{C_{1}}{A_{1}^{2}+B_{1}^{2}} \frac{\beta^{*}-\beta_{c}^{*}}{\beta_{c}^{*}},
$$

where $C_{1}=\frac{\delta \hat{W}^{\infty}-\delta \hat{W}^{b}}{\Omega_{c}} A_{1}-\omega_{d s} \tau_{w}^{*} \delta \hat{W}^{b} B_{1}, A_{1}=\beta_{c}^{*} \beta P_{c}-$ $\frac{2\left(\delta \hat{W}^{\infty}-\delta \hat{W}^{b}\right)}{\Omega_{c}^{3} \omega_{d s}^{2} \tau_{w}^{* 2}}$

$$
\begin{aligned}
& B_{1}=\beta_{c}^{*} \beta S_{c}-\frac{\delta \hat{W}^{\infty}-\delta \hat{W}^{b}}{\Omega_{c}^{2} \omega_{d s} \tau_{w}^{*}}, \\
& P_{c}=\operatorname{Re}\left[\left.\frac{\partial}{\partial \Omega}\left(\frac{\delta \hat{W}_{K 0}}{\beta_{h}}\right)\right|_{\Omega_{c}}\right] \\
& S_{c}=\operatorname{Im}\left[\left.\frac{\partial}{\partial \Omega}\left(\frac{\delta \hat{W}_{K 0}}{\beta_{h}}\right)\right|_{\Omega_{c}}\right] .
\end{aligned}
$$

Equation (12) also theoretically indicates possible conditions for the existence of an unstable FLM branch as $\beta^{*}>\beta_{c}^{*}$, for instance, when $C_{1}>0$. The calculation shows that $C_{1}(=0.025)$ has a positive sign for the case studied in this Letter. The calculated growth rate from Eq. (12) [dash-dotted curve in Fig. 1(a)] agrees well with the exact numerical solution of Eq. (11) [dashed curve in Fig. 1(a)] near the marginal point, confirming the correctness of the numerical solution.
In conclusion, when the perpendicular beta $\beta^{*}$ of the trapped EPs exceeds a critical value $\beta_{c}^{*}$, the FLM instability with external kink eigenstructure can be triggered, which rapidly grows with increasing $\beta^{*}$ ( $>\beta_{c}^{*}$ ), and eventually becomes the dominant instability. Moreover, the FLM real frequency is in the range of the magnetic precession frequency of trapped EPs. The above characteristics of the FLM are a qualitatively consistent experimental observation [11]. The dominant kinetic contribution from the EPs, for the FLM, comes from the resonance term $\ln [(1+\sqrt{\Omega}) /(1-\sqrt{\Omega})]$, while the dominant resonance term in the $q=1$ fishbone normally comes from the term $\ln (1-1 / \Omega)[14,15]$. Both resonances are associated with the magnetic precession of fast ions, but occurring in different regimes. In addition, the conventional RWM branch is stabilized by the mode resonance with EPs in our model, and potentially also by other damping mechanisms (e.g., the Alfvén and sound wave continuum damping in the presence of plasma flow, the mode resonance with thermal particles, etc.). Finally, we point out that in certain ranges of the plasma rotation speed and the hot ion beta, a mode conversion can occur between the RWM and FLM.

G. Z.H. thanks Dr. H. B. Jiang and Dr. H.D. He for helpful discussions. Y. Q. L. acknowledges very useful discussions with Dr. R. J. Hastie. This work was supported by the National Natural Science Foundation of China under Grant No. 10775040 and also supported in part by the National Magnetic Confinement Fusion Science Program under Grant No. 2009 GB101002.

*Yueqiang.Liu@ccfe.ac.uk

[1] A. Bondeson and D. J. Ward, Phys. Rev. Lett. 72, 2709 (1994).

[2] R. Betti and J. P. Freidberg, Phys. Rev. Lett. 74, 2949 (1995).

[3] M. S. Chu et al., Phys. Plasmas 2, 2236 (1995).

[4] B. Hu and R. Betti, Phys. Rev. Lett. 93, 105002 (2004).

[5] Y. Q. Liu et al., Phys. Plasmas 15, 112503 (2008).

[6] I. T. Chapman et al., Plasma Phys. Controlled Fusion 51, 055015 (2009).

[7] J. W. Berkery et al., Phys. Rev. Lett. 104, 035003 (2010).

[8] Y. Q. Liu, Nucl. Fusion 50, 095008 (2010).

[9] J.W. Berkery et al., Phys. Plasmas 17, 082504 (2010).

[10] Y.Q. Liu et al., Plasma Phys. Controlled Fusion 52, 104002 (2010).

[11] G. Matsunaga et al., Phys. Rev. Lett. 103, 045001 (2009).

[12] M. Okabayashi et al., Nucl. Fusion 49, 125003 (2009).

[13] Y. Q. Liu et al., Phys. Plasmas 15, 092505 (2008).

[14] L. Chen, R. B. White, and M. N. Rosenbluth, Phys. Rev. Lett. 52, 1122 (1984).

[15] R. B. White et al., Phys. Fluids 28, 278 (1985).

[16] R. O. Dendy et al., Phys. Plasmas 2, 1623 (1995).

[17] P. Helander et al., Phys. Plasmas 4, 2181 (1997).

[18] Y. Wu, C.Z. Cheng, and R. B. White, Phys. Plasmas 1, 3369 (1994). 